Exercices Exercices de rhétorique

de rhétorique $\quad 8 \mid 2017$

La rhétorique biblique et sémitique

Le maître et le disciple envoyés à Israël et aux
nations. Analyse rhétorique biblique de Mt 10,1-42

Roberto Di Paolo

Traducteur : Roland Meynet

CpenEdition

Journals

Édition électronique

URL : https://journals.openedition.org/rhetorique/492

DOI : $10.4000 /$ rhetorique.492

ISSN : 2270-6909

Éditeur

UGA Éditions/Université Grenoble Alpes

Édition imprimée

ISBN : 978-2-84310-343-8

Référence électronique

Roberto Di Paolo, « Le maître et le disciple envoyés à Israël et aux nations. Analyse rhétorique biblique de Mt 10,1-42 », Exercices de rhétorique [En ligne], 8 | 2017, mis en ligne le 25 janvier 2017, consulté le 28 novembre 2021. URL : http://journals.openedition.org/rhetorique/492 ; DOI : https://doi.org/ $10.4000 /$ rhetorique.492

Ce document a été généré automatiquement le 28 novembre 2021.

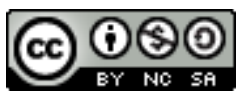

Les contenus de la revue Exercices de rhétorique sont mis à disposition selon les termes de la Licence Creative Commons Attribution - Pas d'Utilisation Commerciale - Partage dans les Mêmes Conditions 4.0 International. 


\title{
Le maître et le disciple envoyés à Israël et aux nations. Analyse rhétorique biblique de Mt 10,1-42
}

\author{
Roberto Di Paolo \\ Traduction : Roland Meynet
}

\section{NOTE DE L'ÉDITEUR}

Pour agrandir les images, cliquer en-dessous sur les mentions « Agrandir » (taille moyenne) ou «Original »(taille réelle).

\section{Introduction}

1 Il est incontestable que le chapitre 10 de Matthieu constitue une unité au sein du récit de l'Évangile. Il est cependant difficile de trouver une structure admise par les auteurs qui ont tenté d'en dégager la composition ${ }^{1}$. Parmi eux on peut citer Ulrich Luz ${ }^{2}$, William D. Davies - Dale C. Allison ${ }^{3}$, Jean Radermakers ${ }^{4}$, Donald Hagner ${ }^{5}$, Massimo Grilli Cordula Langner ${ }^{6}$, Rinaldo Fabris ${ }^{7}$, Schuyler Brown ${ }^{8}$, Dorothy Jean Weaver ${ }^{9}$, Joachim Gnilka ${ }^{10}$, Craig S. Keener ${ }^{11}$, Santi Grasso ${ }^{12}$. Ces auteurs réagissent de manière semblable au problème de la composition du texte. Pour 10,1-23 ils proposent à peu près la même structure; en revanche pour 24-42 ils offrent des structures différentes en fonction de la perspective adoptée dans leur travail et des thèmes qu'ils privilégient dans le texte. De telles différences suffisent à donner une idée de la complexité de ce chapitre de Matthieu.

2 L'apport de l'analyse rhétorique biblique peut aider à découvrir une composition cohérente en elle-même et à l'intérieur du récit matthéen : tel est le but du présent 
travail, qui part de l'hypothèse que le chapitre 10 constitue une séquence et qui se concentrera sur la composition ${ }^{13}$, laissant donc de côté les questions textuelles (critique textuelle, questions grammaticales, lexicographie), le contexte biblique et jusqu'à l'interprétation, qui constitue le point d'arrivée de toute analyse exégétique.

Introduite par un passage narratif (1-5b), la séquence de Mt 10,1-42 comprend cinq passages articulés en trois sous-séquences :

\begin{tabular}{l}
\hline L'envoi des DOUZE \\
\begin{tabular}{|lc|}
\hline Les DOUZE envoyés aux brebis perdues & $1-5 \mathrm{~b}$ \\
\hline Les DOUZE envoyés comme brebis, serpents et colombes & $5 \mathrm{c}-15$ \\
\hline & $16-23$ \\
\hline Le DISCIPLE envoyé est comme son MAITRE & $24-25$ \\
\hline La peur et la confiance des envoyés & $26-31$ \\
\hline L'envoyé digne de son MAITRE & $32-42$ \\
\hline
\end{tabular}
\end{tabular}

\section{0 . Introduction narrative : l'envoi des Douze (10,1-5b)}

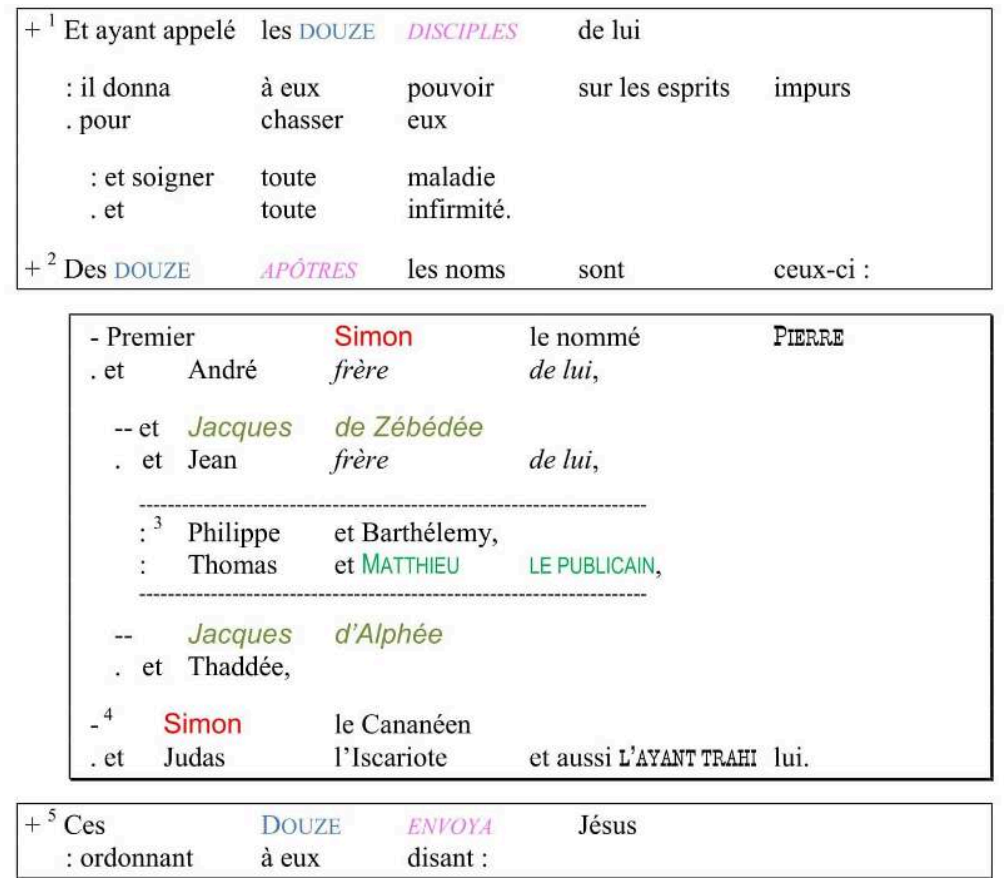

4 Aux extrémités de la première partie, les « douze disciples » (1a) deviennent « apôtres » (2a), c'est-à-dire « envoyés » (apostolous) comme le dit le premier verbe de la dernière partie qui est de même racine (apostellō : 5a). 
5 Dans la liste des Douze, ce sont d'abord deux couples de frères (2bc.2de); dans le dernier morceau se trouvent aussi quatre noms, dont un autre «Jacques» et un autre "Simon ", distingués de ceux du premier morceau par un complément. On peut dire qu'aux extrémités Simon et Judas s'opposent par leurs qualificatifs de «Pierre » et de «le trahissant ». Dans le morceau central, quatre autres noms dont le dernier est celui de Matthieu, l'auteur de l'évangile.

\section{La première sous-séquence du discours $(5 c-23)$}

Cette sous-séquence comprend deux passages $(5 c-15 ; 16-23)$.

\subsection{Les Douze sont envoyés aux brebis perdues (5c-15)}

7 Étant donnée la complexité du passage, il est nécessaire d'analyser d'abord la composition de chacune de ses trois parties avant de présenter l'ensemble.

Première partie $(5 c-8 d)$

\begin{tabular}{|c|c|c|c|}
\hline $\begin{array}{l}-{ }^{5 \mathrm{c}} \text { Sur la route } \\
- \text { et dans une ville }\end{array}$ & $\begin{array}{l}\text { des païens } \\
\text { de Samaritains }\end{array}$ & $\begin{array}{l}\text { n'allez pas } \\
\text { n'entrez pas ; }\end{array}$ & \\
\hline $\begin{array}{l}+{ }^{6} \text { mais partez } \\
+ \text { vers les brebis }\end{array}$ & $\begin{array}{l}\text { plutôt } \\
\text { perdues }\end{array}$ & de la maison & d'Israël. \\
\hline $\begin{array}{l}::{ }^{7} \text { Or partant } \\
:: \text { « S'est approch }\end{array}$ & $\begin{array}{l}\text { proclamez } \\
\text { le règne }\end{array}$ & $\begin{array}{l}\text { disant que } \\
\text { des Cieux. » }\end{array}$ & \\
\hline$:{ }^{8}$ Les infirmes & soignez, & & \\
\hline $\begin{array}{l}\text { - les morts } \\
\text { - les lépreux }\end{array}$ & $\begin{array}{l}\text { ressuscitez, } \\
\text { purifiez, }\end{array}$ & & \\
\hline : les démons & chassez. & & \\
\hline
\end{tabular}

8 C'est Jésus qui parle tout au long de la partie, mais la partie est focalisée sur les paroles qu'il met dans la bouche des disciples (7b). Après avoir donné aux Douze l'ordre de « partir » (6a), Jésus précise, au centre de la partie qu'en réalité leur " partir » a déjà été précédé par «le règne des Cieux » qui « s'est approché ». Le rapport entre « les brebis perdues » du premier morceau et tous ceux dont parle le dernier morceau ne semble pas d'identité mais de complémentarité : les Douze devront non seulement "soigner " mais aussi «enseigner» (selon le couple canonique qui exprime la double face de l'activité de Jésus), c'est-à-dire faire revenir dans le droit chemin ceux qui se sont "égarés " (voir la parabole de la brebis perdue: Mt 18,12 sqq); en d'autres termes « appeler au repentir» («Repentez-vous, car le Royaume des Cieux est tout proche»: $3,1 ; 4,17)$. 
Deuxième partie (8e-10)

\begin{tabular}{|c|c|c|}
\hline $\begin{array}{l}+{ }^{8 \mathrm{e}} \text { Gratuitement } \\
=\text { gratuitement }\end{array}$ & $\begin{array}{l}\text { vous avez reçu, } \\
\text { donnez. }\end{array}$ & \\
\hline \multicolumn{3}{|l|}{$={ }^{9}$ Ne gagnez pas } \\
\hline $\begin{array}{l}\text { :or } \\
\quad \text { - pour les ceintures }\end{array}$ & $\begin{array}{l}\text { ni argent } \\
\text { de vous, }\end{array}$ & ni bronze \\
\hline $\begin{array}{l}-{ }^{10} \text { ni sac } \\
\text { ni deux tuniques }\end{array}$ & $\begin{array}{l}\text { pour la route } \\
\text { ni sandales }\end{array}$ & ni bâton. \\
\hline $\begin{array}{l}\text { + Digne en effet } \\
+ \text { de la nourriture }\end{array}$ & $\begin{array}{l}\text { l'ouvrier } \\
\text { de lui. }\end{array}$ & \\
\hline
\end{tabular}

9 Aux deux membres initiaux correspondent, en ordre inverse, les deux morceaux de la seconde sous-partie. Ceux qui ont «reçu» "gratuitement»(8e), recevront leur «nourriture » de ceux à qui ils sont envoyés (10cd) ; "gratuitement donnez » est explicité par le long morceau suivant (9-10b) qui traite du désintéressement de l'apôtre qui ne cherche pas à se faire offrir de l'argent ou d'autres biens et s'abandonne à la générosité d'autrui.

\section{Troisième partie (11-15)}

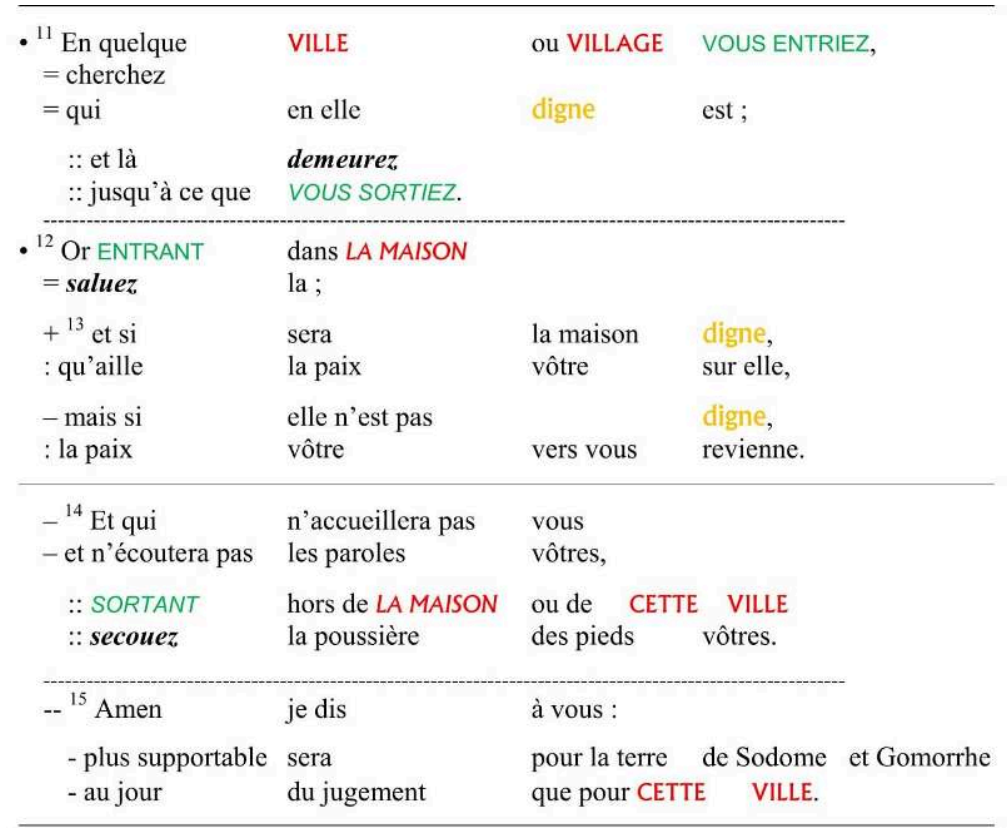

Dans la première sous-partie une double recommandation est donnée, qui regarde l'agglomération, «ville ou village » (11), puis « la maison » qui serait « digne » (12-13) ou non; dans le second morceau est présentée la double éventualité de l'accueil (13ab) ou du refus (13cd). La deuxième sous-partie n'envisage plus que le refus (14), qui sera sanctionné plus sévèrement que pour les pires villes, celles qui n'ont pas respecté les envoyés de Dieu (Gn 19). 


\section{L'ensemble du passage $(10,5 c-15)$}

${ }^{5 c}$ N'allez pas sur la route des païens et $N^{\prime}$ ENTREZ PAS dans une VILLE des Samaritains;
${ }^{6}$ partez plutôt vers les brebis perdues de la MAISON d'Israël. ${ }^{7}$ Or partant, proclamez en
disant : « Le règne des Cieux s'est approché ». ${ }^{8}$ Soignez les infirmes, ressuscitez les
morts, purifiez les lépreux, chassez les démons.

${ }^{8 b}$ Vous avez reçu gratuitement, donnez gratuitement. ${ }^{9} \mathrm{Ne}$ prenez ni or, ni argent, ni (monnaie de) cuivre dans vos ceintures, ${ }^{10} \mathrm{ni}$ sac de route, ni deux tuniques, ni sandales, ni bâton, car l'ouvrier est digne de sa nourriture.

\footnotetext{
${ }^{11}$ Dans quelque VILLE ou village où VOUS ENTRIEZ, cherchez qui y est digne ; et demeurez là jusqu'à ce que vous sortiez. ${ }^{12}$ ENTRANT dans la MAISON saluez-la ; ${ }^{13}$ et si la MASON est digne, que votre paix aille sur elle, mais si elle n'est pas digne, que votre paix revienne sur vous.

${ }^{14}$ Et qui ne vous accueillera pas et n'écoutera pas vos paroles, sortant hors de cette MAISON ou de cette VILLE, secouez la poussière de vos pieds. ${ }^{15}$ Amen je vous le dis, ce sera plus supportable pour la terre de Sodome et Gomorrhe au jour du jugement que pour cette VILLE.
}

11 Les occurrences de "entrer" (5c.11-12) jouent le rôle de termes initiaux pour les passages extrêmes ; « une ville des Samaritains » et « la terre de Sodome et Gomorrhe » peuvent être considérées termes extrêmes, car elles ont en commun d'être des étrangers.

Les passages extrêmes traitent de la prédication (7), soit des " paroles » adressées à ses destinataires (14), tandis que le passage central précise les conditions de l'annonce. Ainsi tout est focalisé sur la gratuité réciproque de l'évangélisation. La dignité est aussi réciproque (1.11.12.13).

On pourra remarquer que la liste des destinataires de la prédication va de l'universel au particulier : « maison d'Israël » (6), « ville » (11.14.15), « maison » (12.13.14).

\subsection{Les Douze envoyés comme brebis, serpents et colombes}

(16-23)

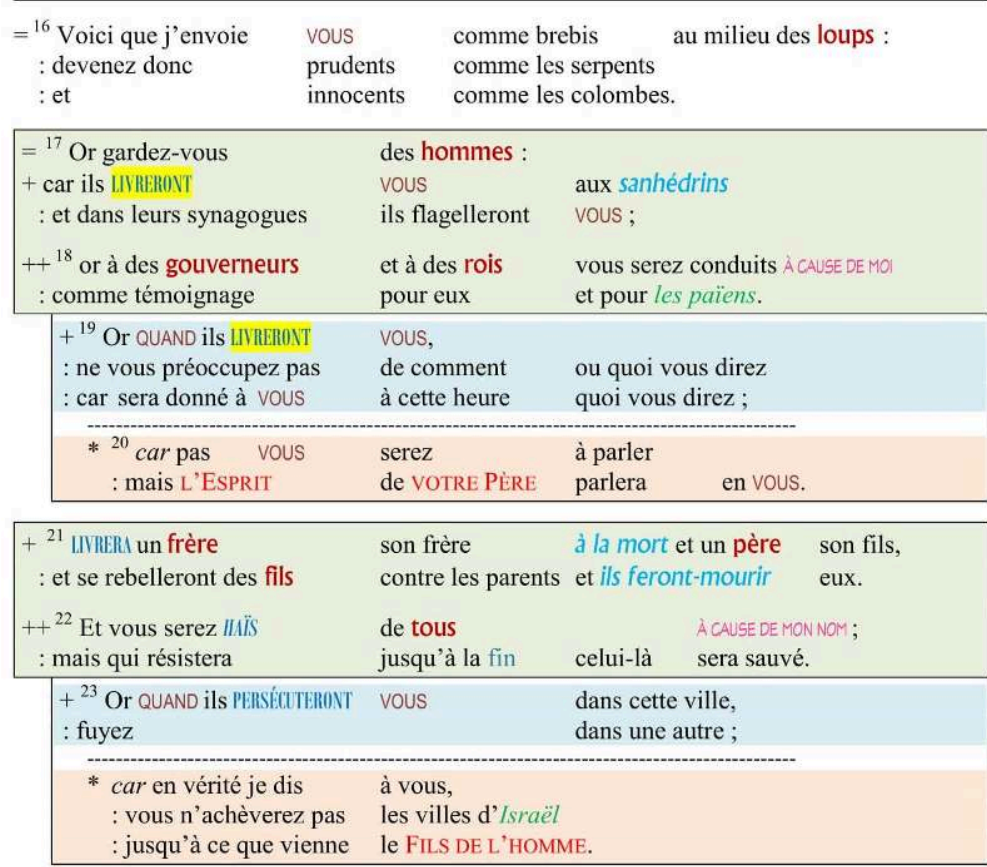


14 La première partie (16) est très brève. Plus développées, les deux suivantes (17-20 ; 21-23) sont parallèles entre elles. Dans les premières sous-parties (17-18; 21-22) la persécution est annoncée, venant des juifs (17) mais aussi des païens (18), des membres de la même famille (21) mais aussi de «tous »; la persécution est toujours « à cause de » Jésus, les deux expressions se trouvant en position symétrique (18a.22a). Dans les sousparties suivantes sont donnés des conseils (19.23ab), dont est fournie enfin la motivation (20.23cde). "L'Esprit de votre Père » et " le Fils de l'homme » peuvent être considérés comme termes finaux des deux dernières parties (20b.23e). Le témoignage vise aussi bien « les païens » qu'« Israël » (18b.23d).

Les «loups» de la première partie (16a) sont les persécuteurs des deux parties suivantes. La prudence des serpents annonce peut-être le conseil de fuir en 23 , et l'innocence des colombes pourrait annoncer l'abandon conseillé en 19.

\subsection{L'ensemble de la première sous-séquence $(5 c-23)$}

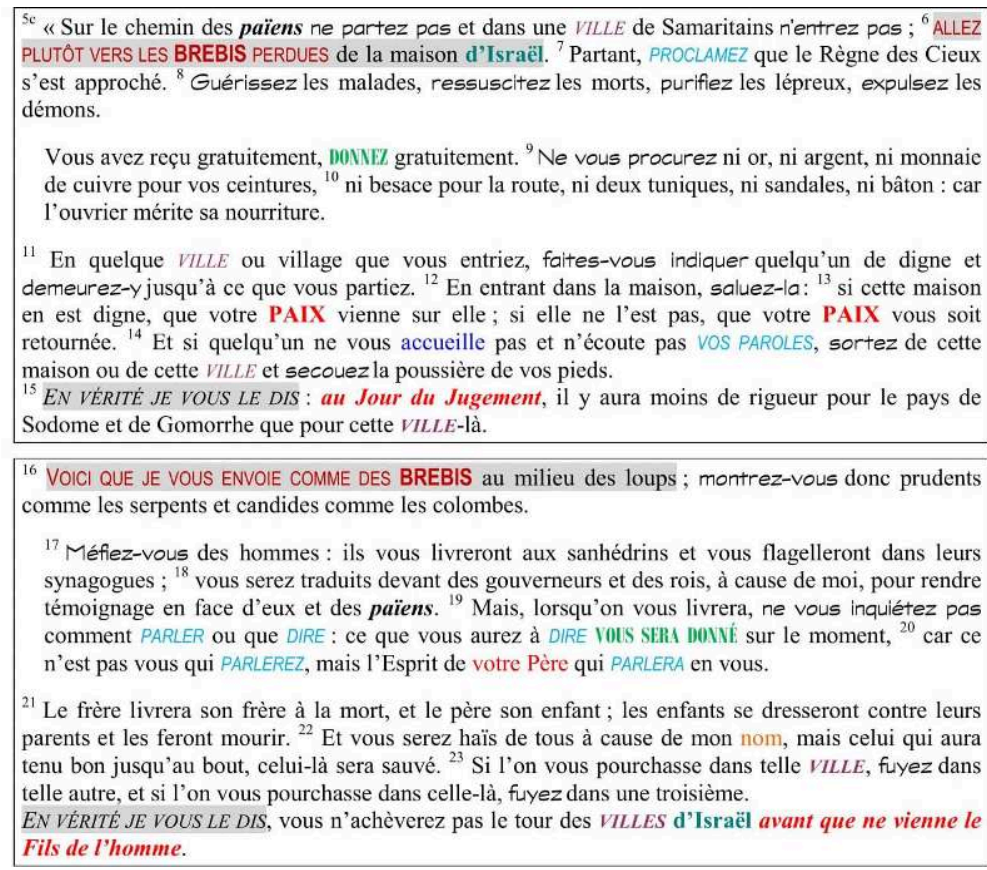

16 Les deux passages s'ouvrent avec deux affirmations complémentaires : ce ne sont pas seulement les destinataires de la proclamation qui sont qualifiés de «brebis » (6), les envoyés le sont aussi (16). Les premières sont " perdues »; étant envoyées " au milieu des loups", les autres se trouveront donc en grand danger d'être perdues elles aussi. Un autre point commun entre envoyés et destinataires est que la parole leur est « donnée », donnée " gratuitement » par les envoyés (8b), donnée aussi gratuitement par l'Esprit aux envoyés quand ils seront persécutés (19). Les deux passages sont parsemés d'impératifs négatifs $(5 \mathrm{c}$ bis. $9 ; 19)$ et positifs (8 quater.11a.11b.12.14a.14b; 16.17.23a.23b).

17 Les deux passages s'achèvent avec une déclaration introduite par la même formule d'insistance (15a.23c) qui regarde le même évènement eschatologique, «au jour du jugement », lors de la venue du « Fils de l'homme ». 


\section{La sous-séquence centrale du discours (24-25)}

18 De la taille d'une partie, le passage forme à lui seul une sous-séquence ${ }^{14}$.

\begin{tabular}{|c|c|c|c|}
\hline $\begin{array}{l}-{ }^{24} \mathrm{~N}^{\prime} \text { est pas } \\
-\mathrm{ni}\end{array}$ & $\begin{array}{l}\text { le DISCIPLE } \\
\text { le SERVITEUR }\end{array}$ & $\begin{array}{l}\text { au-dessus du MATRE } \\
\text { au-dessus du SEIGNEUR }\end{array}$ & de lui \\
\hline$+{ }^{25}$ Il suffit au DISCIPLI & $\begin{array}{l}\text { qu'il devienne } \\
\text { et au SERVITEUR }\end{array}$ & $\begin{array}{ll}\text { comme } & \text { le MAITRE } \\
\text { comme } & \text { le SEIGNEUR }\end{array}$ & $\begin{array}{l}\text { de lui } \\
\text { de lui. }\end{array}$ \\
\hline $\begin{array}{l}\because: \text { Si le MATRE-DE-MASON } \\
\because: \text { combien }\end{array}$ & $\begin{array}{l}\text { Béelzéboul } \\
\text { davantage }\end{array}$ & $\begin{array}{l}\text { ils ont appelé, } \\
\text { LA MAISONNEEEE }\end{array}$ & de lui. \\
\hline
\end{tabular}

19 Dans le premier segment, la relation entre «disciple " et "maître » (didaskalos, "enseignant», 24a) est ensuite exprimée avec l'image du «serviteur " et de son « seigneur » (kyrios, 24b) ; ces mêmes termes sont repris identiquement dans le segment central, tandis que dans le segment final ce sont encore d'autres termes qui sont utilisés : «maître-de-maison » (oikodespotēs, 25d) et "maisonnée » (le pluriel oikiakoi, litt. "gens de la maison", 25e). Dans les deux premiers segments les termes de comparaison, " pas au-dessus » et "comme », sont équivalents; dans le troisième au contraire "combien davantage » indique que la persécution sera bien pire pour les disciples que pour leur maître. Il y a donc progression d'un segment à l'autre.

\section{La troisième sous-séquence du discours (26-42)}

Cette sous-séquence comprend deux passages : 10,26-31;10,32-42.

\subsection{La peur et la confiance des envoyés (26-31)}

\begin{tabular}{|c|c|c|}
\hline \multicolumn{2}{|c|}{ - ${ }^{26}$ NE LES CRAIGNEZ DONC PAS ! } & \multirow{3}{*}{$\begin{array}{l}\text { voilé } \\
\text { sera dévoilé, } \\
\text { caché } \\
\text { sera connu. }\end{array}$} \\
\hline $\begin{array}{l}\text { - Rien, en effet, } \\
\text { : qui ne }\end{array}$ & n'est & \\
\hline $\begin{array}{l}\text { - rien de } \\
\quad \text { : qui ne }\end{array}$ & & \\
\hline $\begin{array}{c}-{ }^{27} \text { Ce que je dis } \\
\text { : dites(-le) }\end{array}$ & à vous & $\begin{array}{l}\text { dans la ténèbre, } \\
\text { dans la lumière; }\end{array}$ \\
\hline $\begin{array}{l}\text { - et ce que } \\
\quad \text { : proclamez(-le) }\end{array}$ & $\begin{array}{l}\text { dans l'oreille } \\
\text { sur les toits. }\end{array}$ & vous entendez, \\
\hline
\end{tabular}

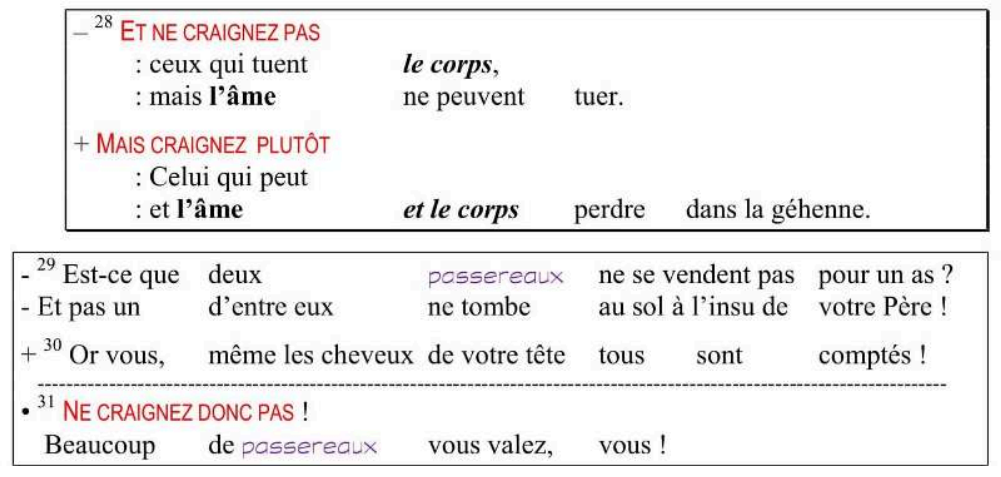

21 Les impératifs du verbe « craindre » se trouvent en termes initiaux des deux segments de la partie centrale (28a.28d) et aux extrémités des première et troisième partie (26a. 31a), faisant ainsi inclusion. 
Dans la première partie, l'invitation à ne pas craindre (26a) est motivée par le second morceau ; celui-ci énonce une vérité générale (26b-e), appliquée ensuite aux disciples (27). Dans la troisième partie l'invitation à ne pas craindre est motivée par le fait que le « Père » céleste prend soin même des passereaux (29), à plus forte raison des disciples (30.31b) ! Le rapport entre les parties extrêmes permet de comprendre que les passifs de 26 sont des passifs divins : si Dieu révèlera ce qui est caché, les disciples ne doivent pas craindre de faire connaître ce qu'ils ont entendu dans l'oreille.

23 La partie centrale (28) oppose les persécuteurs, dont le pouvoir est limité au « corps ", à Dieu capable de sauver l'âme des persécutés et de perdre corps et âme les persécuteurs.

\subsection{L'envoyé digne de son maître (32-42)}

De nouveau, le passage est long et complexe et il faut commencer par analyser ses trois parties avant de présenter l'ensemble.

Première partie (32-36)

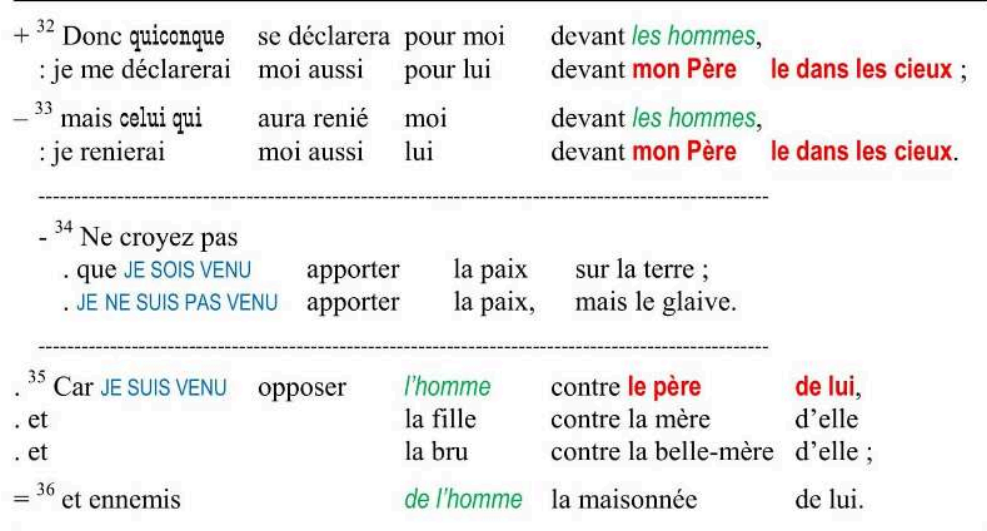

«Le glaive » est ce qui divise et sépare (34). Dans le premier morceau la division qui s'opérera entre ceux qui se déclareront pour Jésus et ceux qui le repousseront se répercutera devant Dieu. Dans le dernier morceau la division qui adviendra entre les membres de la même famille sera due elle aussi à Jésus qui est venu les « opposer ». Le morceau central, plus court, thématise, par opposition, ce pourquoi Jésus « est venu ».

\section{Deuxième partie (37-39)}

\begin{tabular}{|c|c|c|c|}
\hline $\begin{array}{l}-{ }^{37} \text { Qui aime } \\
\quad: n^{\prime} \text { est pas }\end{array}$ & $\begin{array}{l}\text { père } \\
\text { de moi }\end{array}$ & $\begin{array}{l}\text { ou mère } \\
\text { digne }\end{array}$ & plus que $\mathrm{MOI}$ \\
\hline $\begin{array}{l}\text { - et qui aime } \\
: n^{\prime} \text { 'est pas }\end{array}$ & $\begin{array}{l}\text { fils } \\
\text { de moi }\end{array}$ & $\begin{array}{l}\text { ou fille } \\
\text { digne. }\end{array}$ & plus que $\mathrm{MOI}$ \\
\hline $\begin{array}{l}:{ }^{38} \text { Et qui ne prend pas } \\
: \text { et ne suit pas } \\
: \text { n'est pas }\end{array}$ & $\begin{array}{l}\text { la croix } \\
\text { derrière } \\
\text { de moi }\end{array}$ & $\begin{array}{l}\text { de lui } \\
\text { MOI } \\
\text { digne. }\end{array}$ & \\
\hline $\begin{array}{c}-{ }^{39} \text { Qui aura trouvé } \\
\text { : perdra }\end{array}$ & $\begin{array}{l}\text { la vie } \\
\text { elle }\end{array}$ & de lui & \\
\hline $\begin{array}{c}\text { + et qui aura perdu } \\
\text { : trouvera }\end{array}$ & $\begin{array}{l}\text { la vie } \\
\text { elle. }\end{array}$ & de lui & à cause de $\mathrm{MOI}$ \\
\hline
\end{tabular}


26 Ne comprenant qu'un seul segment, le morceau central est encadré par deux morceaux plus développés de rythme semblable.

27 Les deux segments du premier morceau sont complémentaires, le premier indiquant la relation du disciple à ses ascendants, le second à ses descendants : entre les membres les plus proches de sa famille et son maitre le disciple doit décider à qui accorder la priorité. Dans le dernier morceau aussi le disciple doit choisir entre perdre sa vie ou la préserver. Le morceau central énonce le critère du choix : imiter le Christ en portant sa croix.

28 Les cinq occurrences du pronom «qui » jouent le rôle de termes initiaux pour tous les segments.

\section{Troisième partie (40-42)}

29 Chacun des segments du premier morceau est de construction spéculaire (ou chiastique) mais de manière différente, de sorte que la cascade s'enchaîne régulièrement de « vous » à « moi », puis de « moi » à « Celui qui m’a envoyé ».

30 Le second morceau, de construction tout à fait parallèle, distingue parmi les « vous » du premier morceau deux catégories: le "prophète» et le "juste». Celui qui accueille l'envoyé recevra la même récompense que lui.

31 Dans le troisième morceau, "disciple » reprend en généralisant "prophète » et « juste » du morceau précédent, mais le disciple est qualifié de " petit». Le verre d'eau est la plus petite manifestation de l'accueil, qui ne sera pas perdue.

32 Tous les segments, à part le dernier, commencent avec "qui ». Les deux premiers morceaux sont liés par la reprise de "accueillir», les deux derniers par celle de «en son nom de » et de « récompense ${ }^{15} »$.

\begin{tabular}{|c|c|c|c|}
\hline \multirow{3}{*}{$\begin{array}{l}+{ }^{40} \text { Qui accueille } \\
- \text { moi } \\
+ \text { et qui moi } \\
- \text { accueille }\end{array}$} & \multicolumn{3}{|l|}{ vous } \\
\hline & \multicolumn{3}{|l|}{ accueille, } \\
\hline & \multicolumn{3}{|c|}{$\begin{array}{l}\text { accueille } \\
\text { Celui qui a envoyé moi. }\end{array}$} \\
\hline $\begin{array}{l}::{ }^{41} \text { Qui accueille } \\
\quad \text {. une récompense }\end{array}$ & $\begin{array}{l}\text { un prophète } \\
\text { de prophète }\end{array}$ & $\begin{array}{l}\text { EN SON NOM DE } \\
\text { recevra, }\end{array}$ & prophète \\
\hline $\begin{array}{l}:: \text { et qui accueille } \\
\text {. une récompense }\end{array}$ & $\begin{array}{l}\text { un juste } \\
\text { de juste }\end{array}$ & $\begin{array}{l}\text { EN SON NOM DE } \\
\text { recevra. }\end{array}$ & juste \\
\hline $\begin{array}{l}\text { - }{ }^{42} \text { Et qui abreuvera } \\
\text { - d'un breuvage }\end{array}$ & $\begin{array}{l}\text { l'un } \\
\text { d'eau fraîche }\end{array}$ & $\begin{array}{l}\text { de ces } \\
\text { seulement }\end{array}$ & $\begin{array}{l}\text { petits } \\
\text { ENSONNOMDE disciple, }\end{array}$ \\
\hline $\begin{array}{l}: \text { en vérité } \\
: \text { il ne perdra pas }\end{array}$ & $\begin{array}{l}\text { je dis } \\
\text { la récompense }\end{array}$ & $\begin{array}{l}\text { à vous, } \\
\text { de lui. » }\end{array}$ & \\
\hline
\end{tabular}




\section{L'ensemble du passage (32-42)}

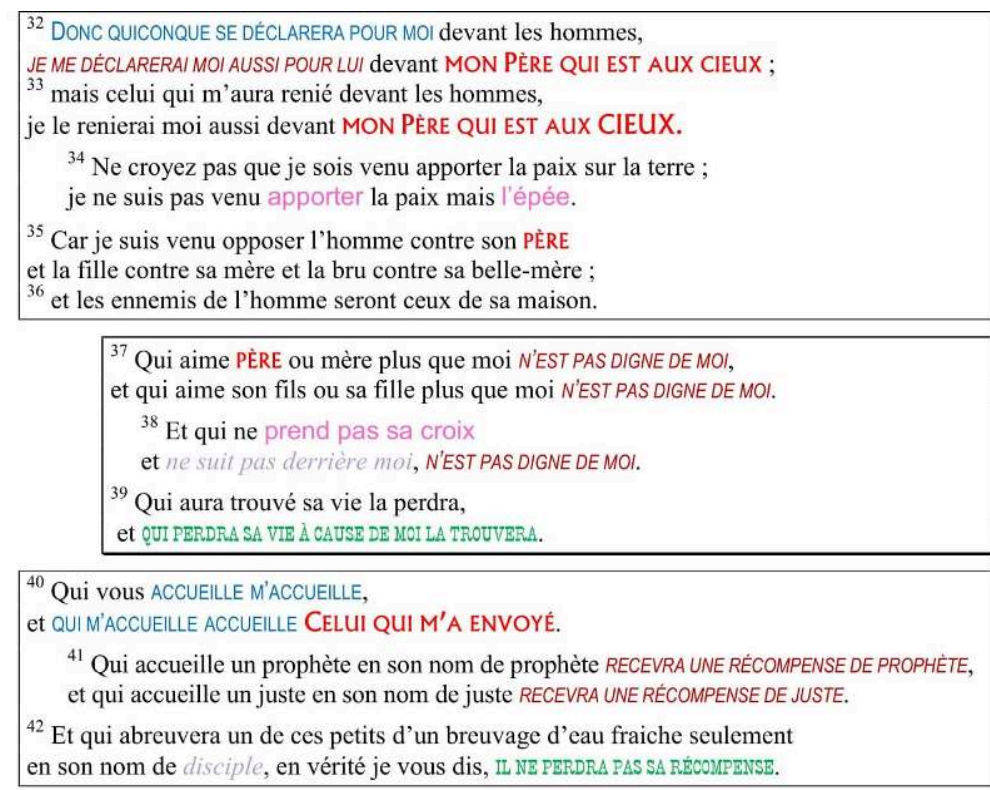
entre les hommes, les disciples, Jésus et Dieu lui-même (32.40); ce dernier est appelé « mon Père qui est aux cieux » (32-33) et «Celui qui m'a envoyé » (40). Puis, tandis que la première partie envisage le cas de celui qui n'accueille pas Jésus (33) et insiste sur la division qu'il apporte (34-36), la dernière partie est entièrement positive, ne considérant que le bon accueil fait aux disciples. (3) centrale articule les deux autres. Le début (37) rappelle la fin de la première partie (35-36) où il est question de la relation de l'homme à son "père "; quant à la fin (39) elle annonce la fin de la dernière partie, où il s'agit de " trouver sa vie » à condition de la « perdre » et de ne pas « perdre » sa récompense.

Les centres des deux premières parties sont en rapport, dans la mesure où " la croix " (38) rappelle « l'épée » (34). On notera en outre que le « disciple » de la fin (42) est celui qui est défini comme celui qui vient derrière son maître au centre (38). 


\subsection{L'ensemble de la troisième sous-séquence (26-42)}
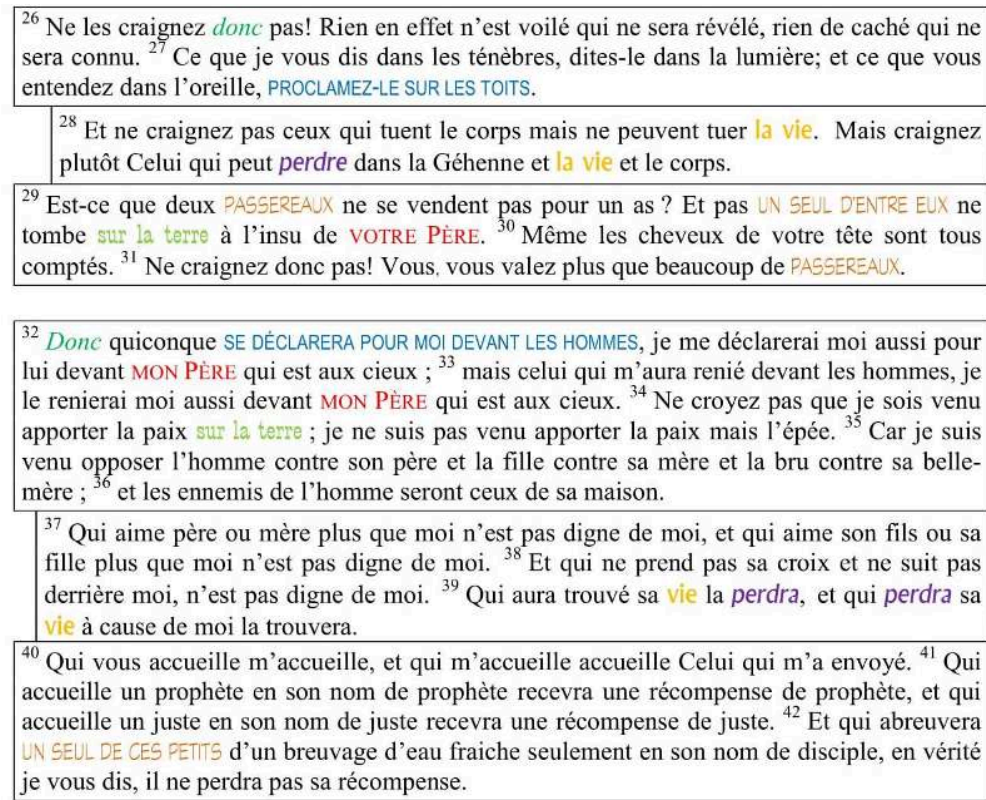

Dans les premières parties, qui commencent également avec «donc» (26.32), « proclamez » correspond à « se déclarera pour moi » (27.32).

Dans les dernières parties, « un seul de ces petits » (42) rappelle « passereaux » et « un seul d'entre eux » (29.31).

Dans les parties centrales $(28 ; 37-39)$, il s'agit de « perdre » ou non sa « vie » (psychē).

Les occurrences de «votre/mon Père » $(29$; 32-33) et de «sur la terre » (29.34) remplissent la fonction de termes médians. 


\section{L'ensemble de la séquence $(10,1-42)^{16}$}

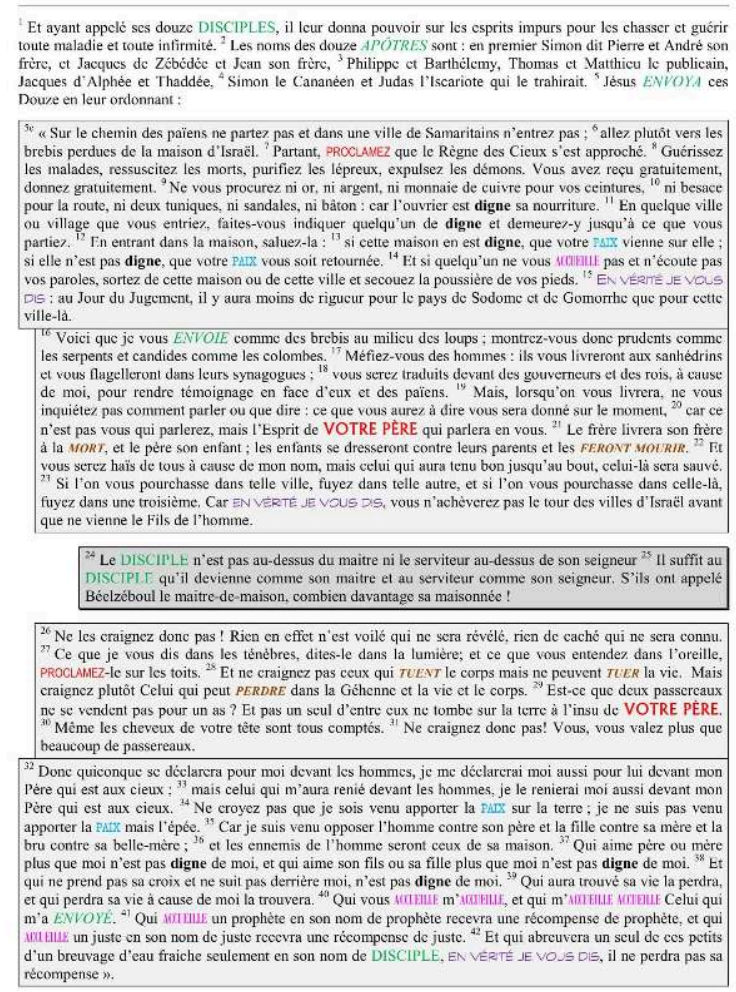

Les quatre occurrences de "disciples» marquent les extrémités et le centre de la séquence $(1.42 ; 24-25)$; ce terme ne se retrouve pas ailleurs dans la séquence. On peut ajouter la cooccurrence du verbe "envoyer " aux extrémités (5.40; et aussi en 16) à quoi il faut ajouter " apôtres » $(2 ;$ litt. » envoyés »).

Les formules d'insistance « En vérité je vous dis » jouent le rôle de termes finaux : non seulement pour les deux passages de la sous-séquence $5 c-23$, comme on l'a déjà noté (15.23), mais aussi pour les deux sous-séquences extrêmes du discours (23.42). Les deux occurrences de " proclamer» (7.27) remplissent la fonction de termes initiaux pour ces deux sous-séquences.

42 La relation entre les quatre passages des sous-séquences extrêmes du discours est de type spéculaire.

- Rapports entre les passages extrêmes $(5 c-15 ; 32-42)$ : «digne» revient quatre fois dans le premier passage (10.11.13 bis), trois dans le dernier (37 bis.38), " paix » est repris deux fois (13 bis; 34 bis), « accueillir» (14) est repris six fois dans le passage final (40-41); enfin les deux occurrences de « en vérité je vous dis » jouent le rôle de termes finaux.

- Rapports entre les passages médians $(16-23 ; 26-31)$ : la persécution va jusqu'à la mort («mort » et «faire-mourir » en 21 ; «tuer » bis et «perdre » en 28$)$, « votre Père » ne revient que dans ces deux passages (20.29).

Le passage central du discours n'a aucun autre mot en commun que « disciple », comme on l'a déjà noté. La ressemblance entre le « disciple » et son « maître » et « seigneur » devrait être la clé de voûte et la clé de lecture de l'ensemble. Il semble que la dernière phrase fasse porter la ressemblance sur le refus et la persécution, qui se retrouve dans chacun des autres passages, surtout dans le passage précédent qui ne traite que de cela, mais déjà à la fin du premier passage du discours (13.14), dans pratiquement tout le passage suivant (28-31), et aussi dans le dernier (34-35). Il faut ajouter que la mention 
de celui qui «le trahirait » à la fin du passage introductif annonçait déjà ce même thème de manière d'autant plus dramatique qu'il s'agissait d'un des douze apôtres.

\section{Conclusion}

Étant donné la longueur du texte analysé et sa complexité d'une part, et les dimensions qui étaient imposées au présent article, il a fallu se limiter à la première étape de l'analyse rhétorique, la composition. Celle-ci n'est que le point de départ qui doit conduire, après la mise en perspective avec d'autres textes bibliques qui peuvent éclairer le discours apostolique de Mt 10 , jusqu'à ce qui représente la fin de tout le travail, à savoir l'interprétation. Celle-ci doit être menée aux différents niveaux d'organisation du texte, depuis le passage, jusqu'à l'ensemble de la séquence, en passant par celui de la sous-séquence. Il faut redire en terminant que si l'étape de la composition n'est que la première, elle n'en représente pas moins les fondations sans lesquelles le reste de la construction ne saurait être vraiment assuré.

L'expérience montre abondamment que l'analyse de la composition sera inévitablement - et heureusement - précisée, complétée, voire corrigée tout au long du parcours des étapes suivantes. C'est dire que la présente étude restera provisoire tant que le travail ne sera pas mené jusqu'à la fin.

\section{NOTES}

1. M. Grilli, dans Comunità e Missione: le direttive di Matteo. Indagine esegetica su Mt 9,35-11,1 (Frankfurt am Main, Peter Lang [Europäische Hochschulschriften, Reihe XXIII, Theologie, Band 458], 1992, p.31-48) étudie à fond les hypothèses de composition de ce chapitre. Déjà H. J. B. Combrink («Structural Analysis of Mt 9:35-11:1», Neotestamentica 11 [1977], p. 111-113) tentait d'étudier le texte comme un ensemble à l'intérieur du premier évangile.

2. U. Luz, Das Evangelium nach Matthäus, Zürich - Neukirchen-Vluyn, Benzinger/Neukirchener (EKK 1/2), 1990 ; trad. anglaise : Matthew 8-20 : A Commentary, Minneapolis MN, Augsburg Fortress Press (Hermeneia), 2001, p. 59-61.

3. W. D. Davies - D. C. Allison, A Critical and Exegetical Commentary on the Gospel according to Saint Matthew, II, Edinburgh, T. \& T. Clark (ICC), 1998, p. 162.

4. J. Radermakers, Au fil de l'évangile selon saint Matthieu, Heverlee, Institut d'Études Théologiques, 1972 ; trad. italienne, Lettura pastorale del vangelo di Matteo, Bologna, Edizioni Dehoniane Bologna (Lettura pastorale della Bibbia 23), 1997, p. 185.

5. D. Hagner, Matthew 1-13, Dallas, Word (Word Biblical Commentary, 33A), 1993, p. 263.

6. M. Grilli - C. Langner, Commentario al Evangelio de Mateo, Estella (Navarra), Verbo Divino (Evangelio y Cultura), 2011, p. 258.

7. R. Fabris, Matteo, Rome, Borla (Commenti biblici), 1982, p. 229.

8. S. Brown, « The Mission to Israel in Matthew's Central Section (Mt 9,35-11,1) », ZNW 69 (1978), p. 76-77.

9. D. J. Weaver, Matthew's Missionary Discourse: A Literary Critical Analysis, Sheffield, JSOT Press (JSNTSup 38), 1990, p. 74. 
10. J. Gnilka, Das Matthäusevangelium, I, Freiburg im Breisgau, Herder, 1986 ; trad. italienne, Il Vangelo di Matteo, Brescia, Paideia (CTNT 1), 1990, p. 525. L'auteur ne présente pas la composition du texte et se contente de présenter ses cinq péricopes : péricope $5-15 ; 16-25 ; 26-33 ; 34-39$; $10,40-11,1$.

11. C. S. Keener, A Commentary on the Gospel of Matthew, Grand Rapids MI - Cambridge UK, William B. Eerdmans, 1999, p. 310. Comme l'auteur précédent, Keener se limite à la présentation de ses six péricopes : péricope $1-4 ; 5-15 ; 16-23 ; 24-33 ; 34-39 ; 40-42$.

12. S. Grasso, Il Vangelo di Matteo. Commento esegetico e teologico, Rome, Città Nuova (Studi Biblici, $36), 2014$, p. 25-26. L'auteur distingue trois parties $(5-15 ; 16-25 ; 26-42)$ articulées en plusieurs sous-unités.

13. Les réécritures donneront à voir la composition, de sorte que la justification de la composition ou sa description sera réduite à l'essentiel.

14. Voir R. Meynet, Traité de Rhétorique biblique, Pendé, Gabalda (Rhétorique sémitique XI), 2013², p. 206.

15. Note du traducteur (R. Meynet): dans le tableau qui suit, de même que le texte biblique est composé, ainsi doit-il en être de la description que l'analyste en fournit. De même que le texte biblique est organisé à plusieurs niveaux, ainsi doit-il en être pour la description de la composition. Pour la partie qui est analysée, la description de chaque morceau est suivie par celle des rapports entre les morceaux. C'est pourquoi la traduction que j'ai donnée de l'original italien a souvent dû être «targoumisante », c'est-à-dire explicitante, et surtout « réorganisante ». (NdE : le targoum est une traduction paraphrastique de la Bible hébraïque en araméen, pour des communautés juives qui ne comprenaient plus l'hébreu.)

16. Note du traducteur (R. Meynet): de nouveau ma traduction a dû être résolument « targoumisante ". Dans le tableau qui suit, les récurrences de mots et surtout de syntagmes sont des marques de composition. Le problème est de déterminer à quels niveaux elles jouent. Pour ce qui est de la présente séquence, on distinguera :

- le niveau de l'ensemble de la séquence, à savoir l'introduction narrative et le discours ;

- le niveau de l'ensemble du discours avec ses trois sous-séquences, la centrale ne comportant qu'un seul passage; où l'on distinguera :

- les rapports entre les sous-séquences extrêmes prises dans leur ensemble $(5 c-23 ; 26-42)$;

- les rapports entre les passages de ces sous-séquences extrêmes, pour voir s'ils se correspondent de manière parallèle ou de manière spéculaire ;

- les rapports du passage central du discours avec les deux sous-séquences qui l'encadrent ;

- enfin les rapports entre l'introduction et le discours.

Erratum : verset 10, lire : « car l'ouvrier est digne de sa nourriture »

\section{AUTEURS}

\section{ROBERTO DI PAOLO}

Directeur de l'Institut supérieur de sciences religieuses G. Toniolo de Pescara (Italie), membre du Conseil directif de la RBS 